\title{
A HOMOLOGICAL METHOD FOR COMPUTING CERTAIN WHITEHEAD PRODUCTS
}

\author{
BY MARTIN ARKOWITZ
}

\author{
Communicated by Norman Steenrod, May 13, 1968
}

1. Introduction. In its simplest form the method for calculating the Whitehead product (WP) $\pi_{n_{1}}(X) \otimes \pi_{n_{2}}(X) \rightarrow \pi_{n_{1}+n_{2}-1}(X)$ may be described as follows. Suppose $X$ is embedded in an $H$-space $E$ so that the pair $(E, X)$ has trivial homotopy groups in dimensions $<n_{1}+n_{2}$. Then we prove that the WP $\left[\alpha_{1}, \alpha_{2}\right]$ of $\alpha_{1} \in \pi_{n_{1}}(X) \equiv \pi_{n_{1}}(E)$ and $\alpha_{2} \in \pi_{n_{2}}(X) \equiv \pi_{n_{2}}(E)$ is the image under a homomorphism $H_{n_{1}+n_{2}}(E)$ $\rightarrow \pi_{n_{1}+n_{2}-1}(X)$ of the Pontrjagin product of $h\left(\alpha_{1}\right)$ and $h\left(\alpha_{2}\right)$ in the homology ring $H_{*}(E)$, where $h: \pi_{*}(E) \rightarrow H_{*}(E)$ denotes the Hurewicz homomorphism. Thus, to determine $\left[\alpha_{1}, \alpha_{2}\right]$, it is necessary to know (1) the effect of $h$ on $\alpha_{1}$ and $\alpha_{2}$, (2) the Pontrjagin product of $h\left(\alpha_{1}\right)$ and $h\left(\alpha_{2}\right)$, (3) the homomorphism $H_{n_{1}+n_{2}}(E) \rightarrow \pi_{n_{1}+n_{2}-1}(X)$.

It is, however, only sometimes possible to find an $H$-space for which the information (1), (2) and (3) is available. As a first example, consider the classifying space $B U_{t}$ of the unitary group $U_{t}$ and the WP

$$
\pi_{2 r+2}\left(B U_{t}\right) \otimes \pi_{28+2}\left(B U_{t}\right) \rightarrow \pi_{2 t+1}\left(B U_{t}\right), t=r+s+1 .
$$

Here we embed $B U_{t}$ in the $H$-space $B U_{\infty}$ and note that the required information is known. In this way we obtain a new proof of a theorem of Bott [1]. For a second example suppose $\pi_{i}(X)=0$ for $i<n$ and $n<i<2 n-1$ and $\pi_{n}(X)=\pi$, where $n$ is odd. Then $X$ can be embedded in $K(\pi, n)$. The Pontrjagin square in $H_{2 n}(\pi, n)$ is zero and so $[\alpha, \alpha]=0$ for any $\alpha \in \pi$. This result is due to Meyer and Stein [8] (see also \$3).

We actually generalize the preceding method by considering $k$ th order WP's instead of ordinary WP's and by requiring that there exist a pair $(E, A)$ with $A$ operating on $E$ rather than an $H$-space $E$. Our main result Theorem 1 then yields for ordinary WP's $(k=2)$ both the assertion of the first paragraph and a theorem of Meyer [4]. For $k>2$ it enables us, in $\$ 3$, to extend Bott's theorem by computing $k$ th order WP's in $\pi_{*}\left(B U_{t}\right)$, and to examine in some detail the $k$ th order WP

$$
\pi_{n}(X) \otimes \cdots \otimes \pi_{n}(X) \rightarrow \pi_{k n-1}(X)
$$

when $\pi_{i}(X)=0$ for $i<n$ and $n<i<k n-1$.

Details of these results and other applications will appear elsewhere. 
We would like to express our thanks to Dartmouth College for the award of a Faculty Fellowship during the time that this work was done. We also acknowledge gratefully several helpful discussions with S. Y. Husseini.

2. The Main Theorem. We recall the definition of a $k$ th order WP [5]. Elements $\alpha_{r} \in \pi_{n_{r}}(X), r=1, \cdots, k$, determine $f: V=S^{n}$ $\bigvee \cdots \bigvee S_{k} \rightarrow X$. Let $T=T\left(S^{n}, \cdots, S^{n}{ }_{k}\right)$ denote the subspace of $P=S^{n} \times \cdots \times S^{n}{ }_{k}$ of $k$-tuples with at least one coordinate at the base point. If

$$
N=\sum_{r=1}^{k} n_{r}, \quad \lambda \in H_{N}(P, T) \approx Z
$$

is a generator and $\hat{f}: T \rightarrow X$ an extension of $f$, then $\hat{f}_{\#} \partial h^{-1}(\lambda)$ is in $\pi_{N-1}(X)$, where $h$ is the Hurewicz homomorphism, $\partial$ the boundary homomorphism and $\hat{f}_{\#}$ the homomorphism induced by $\hat{f}$ :

$$
H_{N}(P, T) \stackrel{h}{\stackrel{\leftarrow}{\approx}} \pi_{N}(P, T) \stackrel{\partial}{\rightarrow} \pi_{N-1}(T) \stackrel{\hat{f}_{\sharp}}{\rightarrow} \pi_{N-1}(X) .
$$

The kth order Whitehead product $\left[\alpha_{1}, \cdots, \alpha_{k}\right]$ is the (possibly empty)

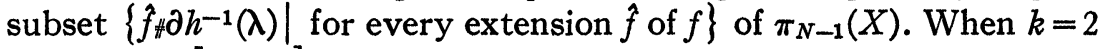
the subset $\left[\alpha_{1}, \alpha_{2}\right]$ consists of a single element, the ordinary WP of $\alpha_{1}$ and $\alpha_{2}$. We say that a subspace $A$ of a space $E$ operates on $E$ if there exists a map $\mu: E \times A \rightarrow E$ such that $\mu \mid E$ is homotopic to the identity map and $\mu \mid A$ is homotopic to the inclusion. Then $\mu$ induces the generalized Pontrjagin product $H_{*}(E) \otimes H_{*}(A) \rightarrow H_{*}(E)$.

Suppose $X$ is 1 -connected and

(a) there exists a pair $(E, A)$ such that $A$ operates on $E$ and the inclusion $i: A \rightarrow E$ induces an isomorphism $i_{\#}: \pi_{\varepsilon}(A) \rightarrow \pi_{s}(E)$ for $s$ $=n_{2}, \cdots, n_{k}$

(b) there exists a map $X \rightarrow E$ such that $\pi_{s}(X) \rightarrow \pi_{s}(E)$ is an isomorphism for $s<N-1$ and an epimorphism for $s=N-1$. By using the mapping cylinder we may assume that the map $X \rightarrow E$ is an inclusion. Then the pair $(E, X)$ is $(N-1)$-connected and so $h: \pi_{N}(E, X) \rightarrow H_{N}(E, X)$ is an isomorphism. Thus a homomorphism $H_{N}(E) \rightarrow \pi_{N-1}(X)$ can be defined as the composition

$$
H_{N}(E) \stackrel{j}{\rightarrow} H_{N}(E, X) \stackrel{h^{-1}}{\approx} \pi_{N}(E, X) \stackrel{\partial}{\rightarrow} \pi_{N-1}(X)
$$

where $j$ is induced by inclusion and $\partial$ is the boundary homomorphism. 
THEOREM 1. Under the assumptions stated above, the kth order WP set

$$
\left[\alpha_{1}, \cdots, \alpha_{k}\right] \text { of } \alpha_{r} \in \pi_{n_{r}}(X), \quad r=1, \cdots, k,
$$

is nonempty and one of its elements is

$$
\partial h^{-1} j\left(h\left(\alpha_{1}\right) * h\left(i_{*}^{-1} \alpha_{2}\right) * \cdots * h\left(i_{*}^{-1} \alpha_{k}\right)\right)
$$

where "*" denotes the generalized Pontrjagin product.

For the next result assume that $X$ is $(p-1)$-connected. Let $X_{n}$ denote the $n$th Postnikov section of $X$ and $X_{q, p+q-2}$ the fibre of $X_{p+q-2} \rightarrow X_{q-1}$. Since this fibration is principal, there is an action of $X_{q, p+q-2}$ on $X_{p+q-2}$. Letting

$$
A=X_{q, p+q-2}, \quad E=X_{p+q-2} \text { and } X \rightarrow X_{p+q-2}
$$

be the projection, we derive Meyer's theorem [4] on the WP of $\alpha_{1} \in \pi_{p}(X)$ and $\alpha_{2} \in \pi_{q}(X)$ :

Corollary 2. $\left[\alpha_{1}, \alpha_{2}\right]=\partial h^{-1} j\left(h\left(\alpha_{1}\right) * h\left(i_{\pi}^{-1} \alpha_{2}\right)\right)$.

We note that $\partial h^{-1} j$ can be identified with the transgression

$$
H_{p+q}\left(X_{p+q-2}\right) \rightarrow H_{p+q-1}\left(F_{p+q-1}\right)=\pi_{p+q-1}(X)
$$

of the fibration

$$
F_{p+q-1} \rightarrow X_{p+q-1} \rightarrow X_{p+q-2} .
$$

Corollary 3. If there exists a map of $X$ into an $H$-space $E$ such that $\pi_{s}(X) \rightarrow \pi_{s}(E)$ is an isomorphism for $s<N-1$ and an epimorphism for $s=N-1$, then $\partial h^{-1} j\left(h \alpha_{1} * \cdots * h \alpha_{k}\right) \in\left[\alpha_{1}, \cdots, \alpha_{k}\right] \subset \pi_{N-1}(X)$, where "*" denotes Pontrjagin product in $H_{*}(E)$.

3. Higher order Whitehead products. Here we use Corollary 3 to calculate some higher order WP's.

THEOREM 4. If $\alpha_{r} \in \pi_{2 m_{r}+2}\left(B U_{t}\right) \approx Z$ and $\gamma \in \pi_{2 t+1}\left(B U_{t}\right) \approx Z_{t !}$ are suitable generators, $r=1, \cdots, k$ and $t=m_{1}+\cdots+m_{k}+k-1$, then

$$
m_{1} ! \cdots m_{k} ! \gamma \in\left[\alpha_{1}, \cdots, \alpha_{k}\right] \subset \pi_{2 t+1}\left(B U_{t}\right) .
$$

The proof proceeds by embedding $B U_{t}$ in $B U_{\infty}$ and applying Corollary 3. The factorials appear because $h\left(\alpha_{r}\right)=m_{r} ! p_{r}$, where $p_{r}$ is a generator of primitive elements in $H_{2 m_{r}+2}\left(B U_{\infty}\right)$ [3].

REMARK. For $k=2$, Theorem 4 provides a new proof of Bott's theorem [1] on the WP $\pi_{2 r+2}\left(B U_{t}\right) \otimes \pi_{2 s+2}\left(B U_{t}\right) \rightarrow \pi_{2 t+1}\left(B U_{t}\right)$ (or, what is the same thing, the Samelson product $\left.\pi_{2 r+1}\left(U_{t}\right) \otimes \pi_{2 s+1}\left(U_{t}\right) \rightarrow \pi_{2 t}\left(U_{t}\right)\right)$, $t=r+s+1$. In addition, we can prove a result similar to Theorem 4 
for the symplectic group $S p_{t}$ and retrieve Bott's theorem on Samelson products in $\pi_{*}\left(\mathrm{~S} p_{t}\right)$ [1].

For the remainder assume that $\pi_{i}(X)=0$ for $i<n$ and $n<i<k n$ $-1(n>1)$ and set $\pi_{n}(X)=\pi$ and $\pi_{k n-1}(X)=G$. Then the $k$ th order WP of elements of $\pi$ is a unique element of $G$. Let $l_{*}: H_{k n}(\pi, n)$ $\rightarrow H_{k n}(G, k n)=G$ be induced by the first Postnikov invariant $l$ of $X$ and denote by $\gamma_{k}: H_{n}(\pi, n)=\pi \rightarrow H_{n k}(\pi, n)$ the $k$ th divided power in the ring $H_{*}(\pi, n)$ [2].

TheOREM 5. Let $\alpha \in \pi_{n}(X)$ and $s_{1}, \cdots, s_{k}$ be any integers.

(a) If $n$ is odd, then $\left[s_{1} \alpha, \cdots, s_{k} \alpha\right]=0$.

(b) If $n$ is even, then $\left[s_{1} \alpha, \cdots, s_{k} \alpha\right]=s_{1} \cdots s_{k} k ! l_{*}\left(\gamma_{k}(\alpha)\right)$.

The proof consists of embedding $X$ in $K(\pi, n)$ and applying Corollary 3. The necessary information on $H_{*}(\pi, n)$ is known [2].

CoRollary 6. In addition, assume that $\pi=G=Z$ and $l=m b^{k}$, a multiple of the kth cup product of the basic class $b \in H_{n}(Z, n)$. Then if $n$ is even, $\left[s_{1} \alpha, \cdots, s_{k} \alpha\right]=m k ! s_{1} \cdots s_{k} \gamma$ for a generator $\gamma$ of $Z$.

Remarks. (1) Porter's result [7] on the $k$ th order WP in complex projective $(k-1)$-space follows immediately from Corollary 6 by setting $n=2$ and $m=1$.

(2) Theorem 5 and Corollary 6 provide another way to obtain some of Porter's examples for certain phenomena regarding higher order WP's [6].

(3) For $k=2$ Theorem 5 is a special case of a theorem of Stein [8]. We note that one direction of Stein's theorem can be extended to $k$ th order WP's.

\section{REFERENCES}

1. R. Bott, $A$ note on the Samelson product in the classical groups, Comment. Math. Helv. 34 (1960), 249-256.

2. Séminaire H. Cartan, 1954-55, Exposés 7 and 11, Secrétariat matématique, Paris.

3. - , 1959-60, Exposé 17. Secrétariat matématique, Paris.

4. J.-P. Meyer, Whitehead products and Postnikov systems, Amer. J. Math. 82 (1960), 271-280.

5. G. J. Porter, Higher order Whitehead products, Topology 3 (1965), 123-135.

6. - Spaces with vanishing Whitehead products, Quart. J. Math. Oxford Ser. (2) 16 (1965), 77-85.

7. - Higher order Whitehead products and Postnikov systems, Illinois J. Math. 11 (1967), 414-416.

8. N. Stein, Note on the realization of Whitehead products, Quart.J. Math. Oxford Ser. (2) 17 (1966), 160-164.

Mathematical Institute, OXford and Dartmouth College 